\title{
Forum
}

\section{Schwerpunktthema: Wie gerecht ist Wählen?}

Wahlen sind ein konstitutives Element von Demokratien. Wahlen dienen regelmäBig der Ermittlung politischen Personals, der Auswahl von Parteien, der Zuweisung von Macht, Ressourcen und Kompetenzen. Jedoch - so zeigt die empirische Wahlforschung - ist die Wahlbeteiligung insbesondere in westlichen Demokratien seit einigen Jahrzehnten rückläufig, beteiligen sich tendenziell immer weniger Bürger daran, ihre politischen Repräsentanten mittels Wahlen zu bestimmen. Die Gründe hierfür sind vielschichtig. Durch die kontinuierlich rückläufige Wahlbeteiligung steigt der Einfluss derjenigen, die zur Wahl gehen, auf die Politik. Im Umkehrschluss sind die Interessen und Anliegen der Nichtwähler tendenziell unterrepräsentiert. Das ist bei einem konstanten prozentualen Anteil der Nichtwähler von 30 Prozent und mehr, in vielen demokratisch verfassten Staaten, eine im Ergebnis für den gesellschaftlichen Zusammenhalt gefährliche Entwicklung.

Auch vor diesem Hintergrund stellt sich die grundsätzliche Frage, wie gerecht Wählen heute eigentlich (noch) ist. Welche Möglichkeiten gibt es, dem Trend der sinkenden Wahlbeteiligung entgegenzuwirken? Welche Alternativen zum Wählen existieren? Wie ist es um deren Legitimität und Praktikabilität bestellt?

Aus diesem Anlass beschäftigt sich das folgende Forum aus dem Blickwinkel unterschiedlicher Teildisziplinen der Politikwissenschaft mit der Frage „Wie gerecht ist Wählen?" Hubertus Buchstein zeigt aus theoretischer Perspektive auf, inwiefern Losen eine gerechtere Alternative zum Wählen darstellt. Thorsten Faas fragt aus dem Blickwinkel der Politischen Systemforschung und mit Bezug auf die Bundesrepublik Deutschland danach, wie gerecht eine Wahlpflicht wäre und lotet deren Möglichkeiten und Grenzen aus. Sebastian Elischer untersucht schließlich aus der international vergleichenden Perspektive, inwieweit Wahlen in Afrika als ein legitimes Verfahren der Auswahl politischen Personals und der Zuweisung von Macht, Ressourcen und Kompetenzen gelten können. 